Ann. Génét. Sél. anim., I970, 2 (3), 325-346.

\title{
VARIABILITÉ GÉNÉTIQUE DU RENDEMENT A L'ABATTAGE ET DE LA COMPOSITION ANATOMIQUE DE LAPINS DE TROIS RACES
}

\author{
R. ROUVIER
}

avec la collaboration technique de J.-P. CAIre, Marie-Claude Scheller, et l'aide de l'Atelier de Calcul du Département de Génétique animale, C.N.R.Z.

\author{
Laboratoive de Méthodologie génétique, \\ Centre de Recherches de Toulouse, 3 I-Auzéville \\ Institut national de la Recherche agronomique
}

\section{RÉSUMÉ}

Sur 160 lapins mâles âgés de $84 \pm 2$ jours, issus de trois races et d'un plan d'accouplement hiérarchisé race-père-mère, nous avons étudié les caractères et variables suivants : poids vif à l'abattage, poids de la peau, du tractus digestif vidé de son contenu, poids de carcasse chaude, poids totaux des tissus musculaire, osseux et gras et de régions ou parties de ces tissus, rapport muscle /os (poids de muscle/poids d'os), rendement à l'abattage, écarts entre les valeurs observées d'un caractère et leurs valeurs estimées par sa droite de régression sur un autre (variables "écarts ").

Les paramètres statistiques des variables étudiées sont donnés pour chacune des trois souches (tabl. 1). Les poids vif d'abattage, de carcasse, du tissu musculaire, de la peau, sont moyennement variables (coefficients de variation $v=12$ à $18 \%$ ). Les poids du tractus digestif et du squelette sont peu variables, celui du tissu gras est très variable. A poids vif constant, la variabilité du poids de carcasse devient faible, celles des poids de la peau et du tractus digestif sont plus élevées. A poids de carcasse constant, le tissu musculaire est très peu variable $(v=2,8$ à $4 \%$ ), le tissu gras est très variable $(v=20$ à $25 \%)$. La variabilité du rapport muscle /os, ou d'une variable écart muscle-os reste importante.

La variabillité de la répartition (tabl. 3 ) des régions du tissu musculaire par rapport à ce dernier est plus grande que celle du tissu musculaire a poids de carcasse constant. Un petit nombre seulement de régions musculaires permettrait d'augmenter la précision de l'estimation du poids total du tissu musculaire dans une équation de régression linéaire multiple faisant déjà intervenir le poids de carcasse. Tous les os considérés apporteraient une information complémentaire significative et de même ordre de grandeur (tabl. 4) pour estimer le poids du tissu osseux dans une équation de régression linéaire faisant déjà intervenir le poids de carcasse.

Les estimations des héritabilités (tabl. 5) des valeurs brutes des poids de peau, du tissu osseux, sont nulles; celles des poids vif et poids de carcasse sont faibles. Un certain nombre d'hypothèses permettent d'expliquer les valeurs élevées de la composante mère de la variance trouvées dans ce cas. Pour les autres caractères, les valeurs trouvées des héritabilités sont moyennes à élevées.

La variabilité génétique additive (tabl. 6) des variables " écarts " permettant d'exprimer la répartition des parties corporelles peau, tractus digestif, carcasse, par rapport au poids vif, est importante. Il en va de même pour les variables exprimant l'importance relative du tissu musculaire par rapport au squelette, ou du tissu gras dans la carcasse. 
Les résultats indiquent qu'on pourrait envisager une sélection efficace pour augmenter le rendement à l'abattage, même à âge et poids vit d'abattaçe constants. De même, il semble possible de réaliser des progrès rapides par sélection, dans la modification de la composition anatomique en augmentant l'importance relative du tissu musculaire par rapport au squelette, même à âge et poids de carcasse constants. Cela pourrait s'effectuer à partir des résultats d'une dissection simplifiée donnant les poids des tissus de certaines régions de la carcasse.

\section{INTRODUCTION}

Il est utile d'envisager les possibilités d'amélioration génétique du rendement à l'abattage et de la composition anatomique du lapin de chair. Un meilleur rendement à l'âge ou au poids d'abattage se traduit, toutes choses égales par ailleurs, par un poids plus élevé de carcasse produite par lapine. Par ailleurs, la composition anatomique des carcasses est un facteur de leur qualité bouchère. Cette qualité correspond, chez le Lapin comme chez les autres mammifères domestiques producteurs de viande, à des pourcentages maximaux, minimaux et optimaux dans les carcasses, respectivement des trois principaux tissus musculaire, osseux et gras.

Nous ne connaissons pas d'études de la variabilité génétique, intra-race, de ces caractères chez le lapin. Les recherches effectuées jusqu'à présent sur le rendement à l'abattage ou la composition anatomique des carcasses concernent leur évolution en fonction de l'âge, la variabilité phénotypique, les différences entre sexes, races et croisements. Ainsi, Shafie et al. (I963), JADWIGA (I963), donnent des indications sur l'évolution du rendement à l'abattage en fonction de l'âge, respectivement chez des lapins de race Giza et Blanc de Vienne. PoDrRASKY (I948) a comparé les rendements à l'abattage de lapins mâles et femelles de poids très variables (60o à $5500 \mathrm{~g}$ ). Les comparaisons des rendements à l'abattage de lapins de plusieurs races et croisements portent sur des effectifs d'animaux mesurés en général très faibles. PALKIN (I946) donne des résultats concernant 6o lapins de 3 races, VILLEGAS (I958) fournit des données de poids de carcasse et poids vif de 36 lapins de 7 races et croisements, FracanzanI (I966) a comparé les rendements à l'abattage de I7 lapins de différentes races et croisements. En ce qui concerne la composition anatomique, CANTIER et al. (rg69) ont étudié 1'allométrie de croissance, entre I semaine et 6 mois d'âge, notamment des trois principaux tissus, dans une souche de lapins de race "commun gris ". Damjanova (I966) compare les pourcentages de gras et d'os chez les mâles et femelles d'une même race à plusieurs âges. PoDHrasky (I948) donne aussi des résultats concernant les poids d'os et de viande. OPIChal (I950) a obtenu des équations linéaires établissant en fonction des poids de carcasse, le pourcentage et le poids de viande, le pourcentage et le poids d'os. LATIMER et SAwIN (I962, I967) ont indiqué des résultats concernant la variation des poids de divers os du squelette chez des lapins adultes dans plusieurs souches. Les données de Piotrowicz (I967) concernent des poids de carcasse, d'os, de gras et de viande, d'animaux provenant de croisements réciproques de deux races. 
Aucun de ces travaux ne fournit des indications sur les paramètres génétiques des caractères considérés et sur les possibilités de leur sélection. Nous avons donc étudié chez le lapin la variabilité génétique et phénotypique, intra et entre races, des poids vifs et de carcasse, de la répartition de parties corporelles, des poids des principaux tissus (os, muscle, gras) et de leur répartition dans la carcasse, dans le but d'en déduire le choix des meilleurs critères de sélection pour modifier le rendement à l'abattage et la composition anatomique. Les premiers résultats, obtenus à partir d'une dissection anatomique détaillée de I6o lapins mâles appartenant à trois races, sont discutés dans le présent mémoire.

\section{MATÉRIEL ET MÉTHODE EXPERIMENTALE}

\section{I. - Matériel animal}

L'étude porte sur 160 lapins mâles répartis comme suit : 58 lapins Fauve de Bourgogne issus de 8 pères et 17 mères, 58 Argenté de Champagne issus de 6 pères et 20 mères et 44 Grand Russe issus de 6 pères et 11 mères. Ills proviennent en général d'une seule portée de chaque mère, la totalité des lapins mâles disponibles d'une portée ayant été mesurés, les femelles ne l'étant pas. Les numéros de portée des lapines varient de 1 à 5 , avec une majorité de numéros 2 et 3 . Les animaux sont nés et ont été élevés dans l'élevage expérimental de la station de génétique quantitative et appliquée, au C.N.R.Z. Les trois souches ont été constituées à partir de reproducteurs acquis de 1963 à 1965 et constituant un échantillon aussi représentatif que possible des trois races. Cependant, du fait de l'effectif limité de reproducteurs utilisés, nous parlerons de souche, les résultats obtenus dans la comparaison de ces trois souches ne pouvant pas se généraliser en s'étendant aux trois races dont elles sont issues. Les lapereaux étaient sevrés à l'âge de 42 jours. Ils étaient ensuite élevés en cage individuelle, sur grillage, jusqu'à l'abattage à l'âge de $84 \pm 2$ jours. Une expérimentation dont les résultats ont été indiqués (Rouvier, 1969) et qui nécessitait la mesure de la quantité d'aliment ingérée par chaque lapin a conduit à adopter ce mode d'élevage en cage individuelle. Dans la période suivant le sevrage, les lapins ont reçu à volonté le même aliment, sous forme de granulé, dosant de 13,4 à 14,7 p. 100 de matière azotée totale et de l'ordre de 14,5 p. 100 de cellulose.

\section{2. - Technique d'abattage}

Les 160 animaux étudiés ont été abattus entre le 19-10-1966 et le 22-03-1967 et disséqués entre le 4-11-1966 et le 24-03-1967. Ils étaient abattus après une diète hydrique de l'ordre de 16 heures. lis étaiekt pesés individuellement lors de la mise à jeun et immédiatement avant l'abattage. La saignée avait lieu par section des vaisseaux sanguins au niveau de la gorge. La peau était enlevée en entraînant la tunique musculaire sous-cutanée et pesée (poids de la peau). La peau recouvrant les mains et les pieds restait en place. La cavité abdominale était ensuite ouverte, l'ensemble du tube digestif (sauf la partie supérieure de l'œesophage), le pancréas, la rate, le tractus uro-génital étant enlevés. Le tube digestif était vidé de son contenu et pesé (poids du tractus digestif). La carcasse chaude restant était pesée dans le quart d'heure suivant la saignée. Láa carcasse était placée dans un réfrigérateur à la température de $+4^{0}$ pendant 24 heures. Elle était ensuite pesée (poids de carcasse froide), enveloppée dans un sac en polystyrène et mise dans un congélateur où elle était conservée à la température de $-18^{\circ} \mathrm{C}$. Les pertes de poids des carcasses, lors du séjour dans le réfrigérateur, représentent en moyenne 5 p. 100 du poids moyen des carcasses chaudes. Ce sont les poids des carcasses chaudes que nous considérerons dans l'analyse des résultats.

\section{3. - Dissection musculaive}

Avant dissection, les carcasses ont été mises à la température ambiante de la salle de dissection. La dissection commençait lorsqu'elles étaient dégelées et pesaient le même poids que lors de la mise dans le congélateur. Lors de la dissection musculaire, les muscles de la partie droite de la carcasse ont été séparés et pesés par régions. Les différentes régions musculaires considérées sont indiquées ci-dessous, la méthode de dissection et les dénominations utilisées étant celles de CantiER et Vezinhet (1968). Ces régions sont numérotées, compte tenu de l'ordre dans lequel les variables correspondantes seront étudiées. 
Muscles du tronc, du cou, du thorax, de l'abdomen

XI I. Région rachidienne, comprenant les muscles long costal, long dorsal, long épineux, multifide dorso lombaire.

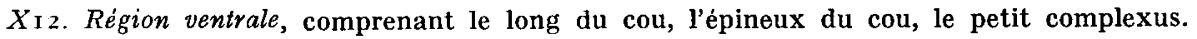

$X$ I3. Région cervicale ventrale et dorsale: le sternocéphalique, le brachiocéphalique, l'omo-trachélien, les sterno-hyoïdien et thyroïdien, le releveur de l'épaule, les scalènes, le trapèze, le rhomboïde, le splénius, le grand complexus.

$X_{\text {I } 4}$. Région dorso-spinale: grand dorsal, le petit dentelé antérieur et postérieur.

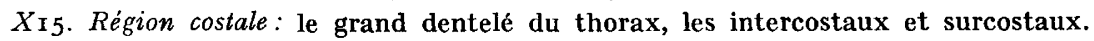

$X I 6$. Région pectorale: les muscles pectoraux.

$X_{\text {I }}$. Région diaphragmatique: les piliers du diaphragme et le diaphragme.

$X \mathrm{I}$. Région abdominale inférieure: les muscles abdominaux.

Xiq. Région sous-lombaire: les muscles sous-lombaires et l'iliaque.

\section{Muscles de l'épaule}

$X$ 20. Région scapulaive: le deltoïde, le sus-épineux, le sous-épineux, le sous-scapulaire, le grand rond, le petit rond, le coraco-brachial.

Muscle du bras et de l'avant-bras: région du bras (X21), région de l'avant bras (X22). Les deux régions indiquées ci-dessus comprennent les muscles décrits par Cantier et VezHINET (1968) dans les paragraphes "bras " et "avant-bras ".

\section{Muscles du bassin}

X23. Région tessière: le fessier superficiel, le fessier moyen, le fessier profond, le pyramidal du bassin, le scansorius.

X24. Région crurale postérieure: le biceps fémoral, le demi-tendineux, le paraméral, le demimembraneux.

$X 25$. Région crurale antérieure: le tenseur du fascia lata, le droit antérieur, le vaste externe, le vaste interne, le vaste intermédiaire.

$X 26$. Région crurale interne: le couturier, le droit interne, le pectiné, l'adducteur.

X27. Région pelvi-crurale profonde: l'obturateur interne et l'obturateur externe, les jumeaux du bassin, le carré crural, le capsulaire de la hanche.

\section{Muscles de la jambe}

X28. Région de la jambe: comprend les muscles décrits par Cantier et Vezhinet (1968) dans le paragraphe "jambe ".

Tous les muscles de la partie gauche de la carcasse ont été pesés ensemble. La somme des poids des muscles des côtés droit et gauche de la carcasse donne le poids du tissu musculaire total $(X 5)$.

\section{4. - Dissection du squelette}

Les os ont été pesés avant et après grattage. Les variables correspondant aux poids des différents os obtenus sont numérotées : bassin $(X 29)$, fémurs $(X 30)$, tibias + péronés $(X 31)$, os des deux pieds + tarses (X32), omoplates + clavicules (X33), humérus (X34), radius + cubitus (X35), os des deux mains + carpes (X36), sternum + côtes + cartilages costaux (X37), colonne vertébrale $(X 38)$, os de la tête $(X 39)$. Le grattage des os, permettant d'enlever des fragments du tissu musculaire, des attaches tendineuses, était effectué manuellement à l'aide d'une curette, sauf pour la tête, la colonne vertébrale, les pieds et les mains. Pour ces derniers, il était effectué par un rat blanc qui mangeait les déchets musculaires les recouvrant, ainsi que les muscles de la tête. Les yeux, la langue et l'encéphale avaient été au préalable enlevés, mais non pesés. Le squelette a été pesé dans sa totalité avant grattage. La somme des poids des os après grattage donne le poids total du squelette (ou du tissu osseux). 


\section{5. - Dissection du tissu adipeux}

La dissection a permis de séparer le tissu adipeux de couverture, interne et intermusculaire. Nous avons obtenu le poids total de ce tissu gras et le poids du gras périrénal (" gras de rognon").

\section{MÉTHODE D'ANALYSE ET RÉSULTATS}

\section{I. - Variables étudiées}

Les variables et caractères étudiés sont les suivants :

$X_{\mathrm{I}}=$ Poids vif

$X_{2}=$ Poids de la peau

$X_{3}=$ Poids du tractus digestif

$X_{4}=$ Poids de carcasse chaude

$X_{5}=$ Poids du tissu musculaire total

$X 6=$ Poids du tissu osseux total

$X_{7}=$ Poids du tissu gras séparable par dissection

$X 8=$ Poids du gras périrénal

$X_{9}=X_{5} / X_{6}:$ Rapport du poids du tissu musculaire au poids du tissu osseux (rapport muscle /os)

$X_{\text {Io }}=X_{4} / X_{\text {I }}:$ Rapport du poids de carcasse au poids vif.

Les variables $X_{\text {I I }}$ à $X_{39}$ (poids de régions musculaires et d'os) ont été énumérées ci-dessus. Nous considérerons de plus des nouvelles variables : certaines correspondent à des caractères que l'on pourrait mesurer à la suite d'une dissection simplifiée des carcasses :

$X_{40}=\left(X 2 \mathrm{I}+X_{22}\right) \times 2=$ Muscles des deux membres antérieurs

$X_{41}=\left(X_{23}+X_{24}+X_{25}+X_{26}+X_{27}+X_{28}\right) \times 2=$ Muscle des deux membres postérieurs et du bassin

$X_{42}=\left(X_{40}+X_{4} \mathrm{I}\right)=$ Muscles des deux membres antérieurs, postérieurs et du bassin

$X_{43}=X_{34}+X_{35}+X_{36}=$ Os des deux membres antérieurs

$X_{44}=X_{29}+X_{30}+X_{31}+X_{32}=$ Os des deux membres postérieurs et du bassin

$X_{45}=X_{43}+X_{44}=$ Os des deux membres antérieurs, des deux membres postérieurs et du bassin.

D'autres variables sont des écarts, comptés parallèlement à l'axe des ordonnées, entre la valeur observée d'un caractère et sa valeur estimée par sa droite de régression sur un autre caractère. Nous appellerons " écarts " ces variables et nous les noterons suivant le principe :

$X_{2}-X_{\mathrm{I}}=$ écart poids de peau - poids vif : poids de la peau $\left(X_{2}\right)$ exprimée 
en écart à sa droite de régression sur le poids vif $(X \mathrm{I})$. Nous considèrerons ainsi les nouvelles variables :

$$
X_{46}=X_{2}-X_{\mathrm{I}} ; X_{47}=X_{3}-X_{\mathrm{I}} ; X_{48}=X_{4}-X_{\mathrm{I}} ; X_{49}=X_{5}-X_{4} ;
$$

$X_{50}=X 6-X_{4} ; \quad X_{51}=X_{7}-X_{4} ; \quad X_{52}=X 8-X_{4} ; \quad X_{53}=X_{5}-X 6 ;$ $X_{54}=X_{40}-X_{43} ; X_{55}=X_{4 \mathrm{I}}-X_{44} ; X_{56}=X_{42}-X_{45}$.

\section{2. - Variabilité phénotypique intra-souche}

Les trois souches seront désignées par $A$ (Fauve de Bourgogne), $B$ (Argenté de Champagne), C (Grand Russe).

Nous avons calculé, séparément pour chaque souche, les moyennes, écartstypes, coefficients de variation $(v)$ des caractères $X \mathrm{I}$ à $X$ Io. Les résultats sont indiqués dans le tableau I. Nous avons également indiqué dans ce tableau les coefficients de variation résiduels $\left(v_{r}\right)$ de variables $y$ considérées comme dépendantes, une variable indépendante $x$ étant maintenue constante. Ceux-ci sont donnés par la formule,

$$
v_{r}=v \sqrt{\mathrm{I}-r^{2}{ }_{x y}}
$$

$r_{x y}$ étant le coefficient de corrélation linéaire entre $x$ et $y$.

Pour les poids de la peau, du tractus digestif, de la carcasse, on a calculé les coefficients de variation résiduels, à partir des écarts-types des écarts des poids observés à ceux estimés par la droite de régression sur le poids vif. Il s'agit de coefficients de variation résiduels à poids vif constant. Ils traduisent la variabilité phénotypique de la répartition des parties corporelles par rapport au poids vif. Pour les poids des trois principaux tissus, du rapport muscle/os, on a calculé les coefficients de variation résiduels à poids de carcasse constant. Ils traduisent la variabilité de la composition anatomique des carcasses.

Les corrélations totales des poids des trois principaux tissus entre eux sont indiquées dans le tableau 2.

Le tableau 3 donne, par souche, les moyennes, coefficients de variation, coefficients de variation résiduels à poids de tissu musculaire constant, corrélations partielles avec le poids total du tissu à poids de carcasse constant, des poids des régions musculaires disséquées. Le tableau 4 donne les résultats homologues pour les poids d'os.

\section{3. - Variabilité génétique intra-souche}

Une analyse de variances et covariances, suivant la hiérarchie souche père - mère - descendants, a été effectuée pour les variables $X_{\mathrm{I}}$ à $X 8, X_{40}$ à $X_{45}$. Dans le tableau 5 , nous indiquons quelles sont les fractions de la variance phénotypique dues à la variance des effets de chaque facteur souche, père, mère, descendant, considérés comme aléatoires. La signification par rapport à o des composantes mère de la variance a été testée. Nous avons considéré, pour effectuer 
ces tests, que le rapport du carré moyen entre mères, intra-père et souche, au carré moyen intra-mère, suivait une loi $F$ de SNEDECOR. Nous indiquons également les valeurs des composantes père de l'héritabilité, estimées intra-souche.

Nous avons mis au point une méthodologie permettant d'étudier, en utilisant les résultats d'analyse hiérarchique des variances et covariances, la variabilité génétique de la répartition des parties corporelles par rapport au poids vif, des tissus par rapport au poids de carcasse. L'importance relative de deux variables a souvent été exprimée, en zootechnie, par leur rapport. Or ces rapports présentent l'inconvénient de conduire à des corrélations artificielles, comme l'a rappelé DiNKEL et al. (I965) (telles que celle entre un rapport et son dénominateur ou son numérateur), et de mal se prêter aux analyses statistiques (voir par exemple, la discussion de RICARD et Rouvier, I967). Nous avons cependant étudié, sur le plan de la variabilité phénotypique, les rapports poids de muscle/poids d'os, et poids de carcasse / poids vif, du fait de leur signification concrète. Mais, de façon générale, dans la présente étude, l'importance relative d'un caractère $(y)$ par rapport à un autre $(x)$, a été exprimée par une nouvelle variable $z$, écart de la valeur observée d'un caractère à sa droite de régression sur l'autre : $z=y-b x+k$ où $k$ est une constante, $b$ est le coefficient de régression de $y$ sur $x$ calculé intra-famille de mère. Il a alors été possible d'obtenir les composantes père, mère, intra-mère, et éventuellement souche, de cette nouvelle variable $z$.

\section{Soient :}

$\sigma_{p_{y}}^{2}$ et $\sigma_{p_{x}}^{2} ; \sigma_{m_{y}}^{2}$ et $\sigma_{m_{x}}^{2} ; \sigma_{e_{y}}^{2}$ et $\sigma^{2} e_{x}$, les variances des effets père, mère, intramère, des deux caractères $y$ et $x$, et soient $\sigma p_{y} p_{x}, \sigma m_{y} m_{x}, \sigma e_{y} e_{x}$ les covariances de ces effets. Nous avons calculé $\sigma_{p_{z}}^{2}, \sigma_{m_{z}}$ et $\sigma_{e_{z}}{ }^{2}$ par les formules de la forme $\sigma_{p z}^{2}=\sigma_{p y}^{2}+b^{2} \sigma_{p_{x}}^{2}-2 b \sigma_{p_{y} p_{x}}$.

Les héritabilités de ces variables $z$, appelées écarts, ont été obtenues par

$$
h_{p_{z}}^{2}=\frac{4 \sigma_{p_{z}}^{2}}{\sigma_{p_{z}}^{2}+\sigma_{m_{z}}^{2}+\sigma^{2} e_{z}}
$$

Dans le tableau 6 , nous indiquons les fractions de la variance phénotypique $\sigma_{z}{ }_{z}$ calculée intra-souche, dues aux composantes mère et père de la variance, ainsi que la composante père de l'héritabilité. Les écarts considérés sont ceux des poids de la peau, du tractus digestif, de carcasse, par rapport au poids vif, des tissus musculaire, osseux et gras, du gras périrénal, par rapport au poids de carcasse, du total muscle par rapport au total os $\left(X_{53}\right)$, des écarts muscle-os des deux membres antérieurs $\left(X_{54}\right)$, postérieurs et bassin $\left(X_{55}\right)$, postérieurs, antérieurs et bassin $\left(X_{56}\right)$.

La variabilité de l'écart muscle-os, a été étudiée à poids de carcasse constant. Soit $t$ le poids de carcasse. Nous avons considéré, de la même façon que la variable $z$ ci-dessus, la variable $q$,

$$
q=z-b^{\prime} t+k^{\prime}
$$

$b^{\prime}$ étant la régression, intra-famille de mère, de $z$ sur $t$, et $k^{\prime}$ une constante. 


\section{4. - I'ariabilité entre souches}

Les tests de comparaison des pentes des droites de régression indiquent que les régressions suivantes peuvent être considérées comme homogènes entre souches : poids de carcasse chaude sur le poids vif, poids des tissus musculaires, osseux et gras sur le poids de carcasse, du rapport muscle/os sur le poids de carcasse, du poids du tissu musculaire sur celui du squelette. Nous avons donc pu effectuer une analyse de covariance, en supposant les distributions normales, pour tester l'égalité chez les trois souches, des poids de carcasse lorsqu'on a ramené par régression ces trois souches au même poids vif moyen, donc à poids vif constant. De même, nous avons testé l'égalité des poids totaux de muscle, os et gras, ainsi que du rapport muscle/os, à poids de carcasse constant, du poids total de muscle à poids du squelette constant. Les résultats des tests de signification ainsi que les valeurs des pentes des droites de régression communes sont indiquées dans le tableau 7 .

\section{DISCUSSION}

\section{I. - Technique de dissection}

Le poids du tissu musculaire n'englobe pas celui de la tunique musculaire sous-cutanée qui a été pesée avec la peau. Il n'englobe pas non plus les muscles de la tête et de la queue (les coccyciens), ni les déchets musculaires obtenus par grattage. Le poids total du tissu musculaire est donc légèrement sous-estimé, d'une quantité que l'on peut apprécier notamment en se référant à une étude préliminaire (résultats non publiés) que nous avions auparavant effectuée sur 6 o lapins mâles de race Fauve de Bourgogne abattus au même âge de $\mathbf{r} 2$ semaines et disséqués muscle à muscle. Chez ces animaux le poids des muscles de la tunique musculaire sous-cutanée représente en moyenne $3,2 \mathrm{p}$. Ioo du poids de la carcasse chaude. Dans l'étude présente, les déchets musculaires obtenus par grattage des os, qui englobent les muscles de la tête et de la queue, représentent suivant les souches, 4 à 6 p. Ioo du poids des carcasses chaudes. Les déchets divers obtenus en cours de dissection, représentent, en moyenne, $2 \mathrm{p}$. Ioo du poids de carcasse chaude. D'autre part, nous observons que le poids des muscles du côté gauche de la carcasse est en moyenne supérieur de 1'ordre de I I à I 8 grammes, suivant les races, à leur poids du côté droit. Cette différence peut s'expliquer du fait que la dissection musculaire plus globale du côté gauche a été plus rapide que celle du côté droit, et que les pertes par évaporation ont peut-être été moins importantes. Toutefois, les pertes totales en cours de dissection sont peu élevées, elles représentent en moyenne I,4 p. roo du poids de la carcasse chaude. La différence entre les poids des muscles des côtés gauche et droit de la carcasse peut également s'expliquer du fait qu'une partie du gras intermusculaire a pu rester englobée entre les muscles dans la dissection du côté gauche. On peut donc consi- 
dérer que le poids total du tissu musculaire, par rapport au poids de la carcasse chaude, est sous-estimé au plus de l'ordre de 7 à 9 p. Ioo.

Les erreurs systématiques éventuelles sur le poids du squelette sont plus difficiles à évaluer. Signalons que le fait d'utiliser un rat blanc pour le grattage de certaines parties du squelette n'a pas permis d'obtenir le gain de temps escompté. Il était en effet nécessaire de surveiller cet animal de façon à ce qu'il ne ronge pas le tissu osseux lui-même. Une certaine erreur aléatoire semble s'être tout de même introduite, ce qui se traduit par une corrélation relativement faible entre les poids totaux des muscles et du squelette. Cette corrélation, calculée intra-race, est ici de 0,70 , alors qu'elle était de 0,88 dans 1'échantillon de 60 lapins Fauve de Bourgogne mentionné précédemment. Par contre, l'erreur doit être faible sur les parties du squelette grattées manuellement, et notamment sur les poids des segments osseux des membres.

\section{2. - Variabilité phénotypique des valeurs brutes des caractères}

TABLEAU I

Moyennes $(\bar{x})$ et coefficients de variation ( $v$ et $v_{r}$ en $\%$ ) pour chacune des trois souches $A, B, C$

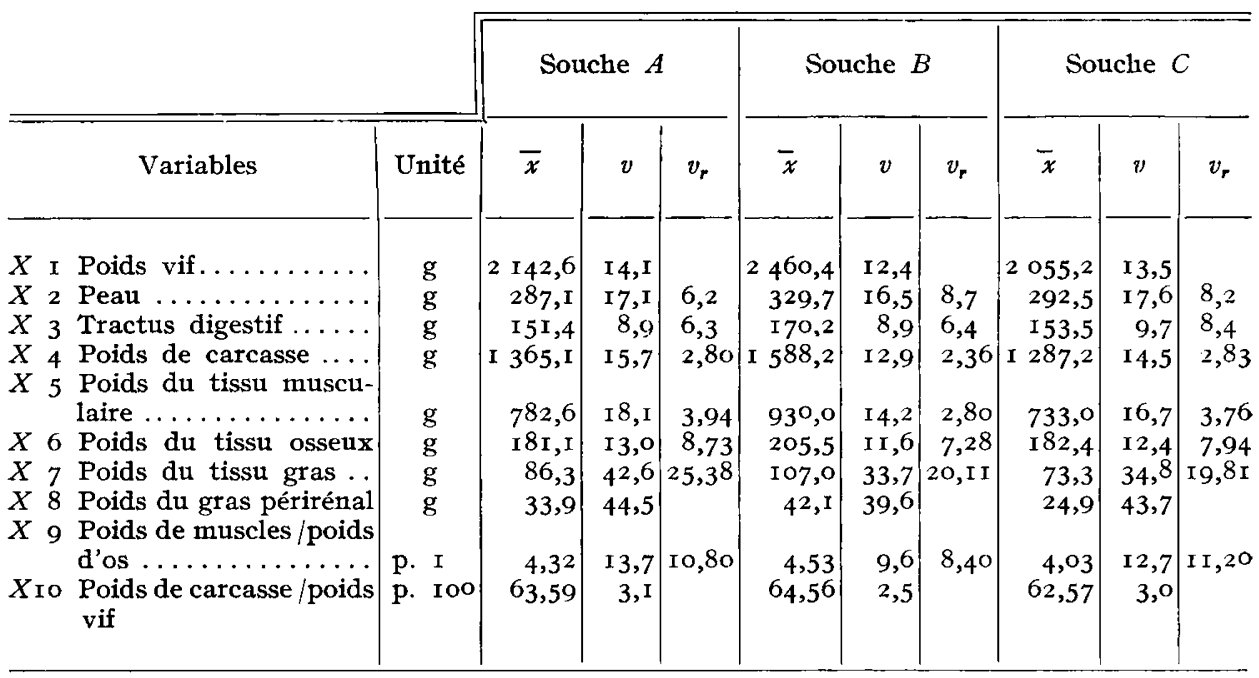

$v_{r}=$ coefficient de variation résiduel, à poids vif constant pour les poids de peau, tractus digestif, carcasse, et à poids de carcasse constant pour les autres caractères.

Les coefficients de variation du poids vif d'abattage ( $v=\mathrm{I} 2$ à I4 \%) et du poids de carcasse $(v=\mathrm{I} 3$ à $\mathrm{I} 6 \%$ ) indiquent que ces deux caractères sont moyennement variables, ainsi que le tissu musculaire $(v=\mathrm{I} 4$ à I $8 \%$ ), la peau $(v=\mathrm{I} 6$ à $\mathrm{I} 8 \%$ ), le tissu osseux ( $v=\mathrm{I} 2$ à I3 \%). Le tractus digestif est peu variable $(v=9$ à ro \%). Le tissu gras est très variable ( $v=34$ à $43 \%$ ). LATIMER et SAwiN, (I947) donnent des coefficients de variation concernant certaines de ces variables 
mesurées chez les lapins mâles et femelles adultes de la race III, race consanguine dérivée de lapins Néo-Zélandais blancs. Chez les mâles, les coefficients de variation du poids vif, du poids de la musculature, du squelette, du tissu gras, correspondent, approximativement en valeur absolue, et exactement dans le classement par ordre de leurs valeurs, à nos résultats. Par contre la variabilité qu'ils trouvent pour 1e poids du tube digestif vide est beaucoup plus élevée $(v=13,7 \%)$. Dans le cas de nos résultats, la variabilité comparée des diverses régions corporelles et des tissus, correspond à leur ordre de développement relatif au cours de la croissance, tel qu'il a été mis en évidence chez le Lapin par CANTIER et al. (I969). Chez les lapins mâles d'une souche de lapins " gris commun " nous avons en effet étudié les croissances relatives, exprimées par leurs coefficients d'allométrie, des tissus par rapport au poids vif vide. Celles du squelette et du tissu musculaire deviennent plus faibles respectivement à partir de 887 -I 569 grammes et de 2242 grammes de poids vif vide. Par contre, celle du tissu gras qui a subi une première augmentation à 887-I 062 grammes devient à nouveau plus élevée à 2 II 7-2 I42 grammes. La variabilité des poids que nous observons ici est d'autant plus grande que les parties corporelles et tissus considérés ont un développement plus tardif.

TABLEAU 2

Corrélations cntre les poids des trois tissus

Ligne I : Souche $A$; Ligne 2 : Souche $B$; Ligne 3 : Souche $C$

Caractères

$X_{5}$. Tissu musculaire

\begin{tabular}{|c|c|c|}
\hline$X_{5}$ & $X 6$ & $X_{7}$ \\
\hline $0,67 \mathrm{I}$ & 0,720 \\
0,744 & 0,770 \\
0,659 & $0,82 \mathrm{I}$ \\
\hline & 0,462 \\
0,480 \\
0,508 \\
\hline
\end{tabular}

$X_{7}$. Tissu gras

Les différences de grandeur des corrélations totales entre les poids des trois tissus correspondent aussi à leur ordre de développement relatif et de variabilité. Les corrélations entre poids des tissus osseux et gras sont plus faibles que celles entre tissus musculaire et osseux, elles-mêmes plus faibles que celles entre tissus musculaire et gras. Nous observions le même ordre des valeurs des corrélations, dans l'échantillon de 60 lapins Fauve de Bourgogne mentionné précédemment, les corrélations étant dans ce cas un peu plus élevées. 


\section{3. - Variabilité phénotypique \\ de la répartition des parties corporelles et des tissus}

Les coefficients de variation résiduels à poids vif constant sont faibles pour le poids de carcasse $\left(v_{r}=2,4\right.$ à $\left.2,8 \%\right)$, un peu plus élevés pour la peau et le tractus digestif vide $\left(v_{r}=6,2\right.$ à $\left.8,4 \%\right)$. La corrélation entre les poids de carcasse chaude et le poids vif est très élevée $(r=0,98)$. L'écart-type, calculé intra-race, du poids de carcasse à poids vif constant reste cependant d'une grandeur notable $(36,4 \mathrm{~g})$. Il peut donc exister des différences importantes entre poids de carcasse d'animaux ayant le même poids vif. Le coefficient de variation du rendement à l'abattage (variable $X$ ro) est légèrement plus élevé que celui du poids de carcasse à poids vif constant. Cela est lié au fait que le rendement dépend du poids vif qui est donc une source de variabilité supplémentaire.

A poids de carcasse constant, le tissu gras est très variable $\left(v_{r}=20\right.$ à $\left.25 \%\right)$, le tissu osseux moyennant variable $\left(v_{r}=\right.$ I2 à $\left.13 \%\right)$, le tissu musculaire peu variable $\left(v_{r}=3\right.$ à $\left.4 \%\right)$. Cet ordre de variabilité des trois tissus correspond au fait que la liaison avec le poids de carcasse est de plus en plus étroite lorsque l'on passe du tissu adipeux au tissu osseux et musculaire. Il est le même que celui observé par Dumonr et al. (r96r) sur 29 châtrons charolais. Au niveau d'un animal, le poids de carcasse ne permet de déterminer qu'avec une faible précision sa composition en ses principaux tissus, à l'exception du tissu musculaire.

Le rapport muscle /os reste moyennement variable, même à poids de carcasse constant $\left(v_{r}=8\right.$ à I I \%). Ce dernier coefficient de variation est plus élevé que ceux des poids des tissus musculaire et osseux, à poids de carcasse constant. Cela correspond aux observations de BERG et BuTTERFIELD (I966) sur bovins; cela confirme l'intérêt pratique du rapport muscle/os pour exprimer la composition anatomique des carcasses, en association avec le poids du tissu gras, tous deux ramenés à poids de carcasse constant. A l'intérieur des trois souches de lapins étudiées ici, ce rapport muscle/os varie dans le même sens que le rendement à l'abattage $(r=0,50$ à 0,56$)$. Il augmente avec le poids de carcasse $(r=0,49$ à 0,63$)$ ou avec celui du tissu gras $(r=0,5 \mathrm{I}$ à 0,55$)$. L'étude des corrélations partielles indique que ces deux caractères semblent jouer sensiblement le même rôle, sur le plan statistique, vis-à-vis du rapport muscle/os. Dans une équation de régression linéaire multiple du rapport muscle/os sur le poids de carcasse et le poids de gras disséqué, l'influence du poids du tissu adipeux ne serait en général pas significative, après prise en compte du poids de carcasse. BERG et BUTTERFIELD (I966) aboutissent aux mêmes conclusions sur bovins. L'augmentation du rapport muscle/os avec le poids de tissu adipeux ou avec le poids de carcasse correspond au fait qu'il y a, intra-souche, plus de tissu adipeux total dans les carcasses plus lourdes. Elle peut aussi traduire le fait qu'il y aurait plus de gras intra-musculaire, pesé avec le muscle, dans les carcasses plus lourdes. Nous avons ajusté le rapport muscle /os au poids de carcasse, plus facile à mesurer que celui du tissu adipeux. Dans l'intervelle des poids considérés, le rapport muscle/os augmente en moyenne, quelle que soit la souche, de $0, \mathrm{I} 4 \pm 0,03$ lorsque le poids de carcasse augmente de roo grammes. L'estimation des valeurs individuelles du rapport muscle/os 
à partir de cette relation est cependant trop imf récise, compte tenu des valeurs des corrélations (tabl. 7). Cette relation indique donc dans quelle mesure le poids de carcasse est en lui-même, pour des animaux d'un poids adulte donné, un critète permettant d'apprécier la qualité bouchère moyenne d'un échantillon de carcasses.

D'un point de vue statistique, le rapport muscle/os doit être soumis aux mêmes critiques que le rendement à l'abattage, et nous ne l'avons considéré ici que du fait de sa signification concrète. Il est préférable d'exprimer la composition anatomique par les poids de chacun des tissus osseux, musculaire et gras, à poids de carcasse constant. Il est de même préférable d'étudier l'écart du poids de muscle observé à celui estimé par sa droite de régression sur le poids d'os, dit écart muscle / os, comme nous l'avons fait pour le Poulet (voir, par exemple Ricard et Rouvrer, I966). Cet écart muscle/os présente des corrélations totales variant, suivant les souches, de 0,58 à 0,65 avec le poids de carcasse, et de 0,55 à 0,65 avec le poids du gras disséqué. Ces deux derniers caractères semblent jouer d'un point de vue statistique approximativement le même rôle. Les corrélations partielles de l'écart muscle /os avec l'un des deux, l'autre étant maintenu constant, sont faibles, une seule sur six étant très significativement supérieure à o. Les conclusions sont donc les mêmes que pour le rapport muscle/os, et ultérieurement nous étudierons l'écart muscle-os à poids de carcasse constant.

\section{4. - Variabilité de la répartition des divers constituants d'un même tissu}

Les coefficients de variation des poids des régions musculaires varient suivant ces régions et les trois souches, de 14,6 p. Ioo à 33, I p. Ioo, avec un maximum de valeurs de 17 à 25 p. Ioo. Les coefficients de variation résiduels sont plus élevés que celui du poids total des muscles à poids de carcasse constant. La répartition du tissu musculaire dans ses diverses régions est deux à trois fois plus variable que celle de la musculature totale sur la carcasse. Cela correspond aux résultats de Dumont et al. (I96I) sur bovins et à ceux de Rouvier et Ricard (I967a) sur poulet. La grande variabilité que nous observons pour certaines régions (pelvicrurale profonde, ventrale, dorso-spinale) dont le poids est d'ailleurs faible, semble liée à la difficulté de leur dissection.

Les corrélations partielles entre poids d'une région musculaire et poids des muscles totaux, à poids de carcasse constant (tabl. 3), permettent de préciser l'intérêt de chaque région pour estimer, par régression linéaire multiple, le poids total des muscles. Cela donne une indication sur les régions musculaires qui seraient à prélever dans une dissection partielle de la carcasse, en vue d'estimer ce poids total du tissu musculaire à partir du poids des muscles d'un petit nombre de régions. A ce point de vue, on note l'intérêt des régions épaule, bras, avant-bras et peut-être jambe, qui pourraient s'obtenir aisément par dissection, sans perte économique importante si la carcasse peut être vendue découpée. D'autres régions sont intéressantes à considérer, mais leur dissection s'accompagnerait d'une dépréciation commerciale de la carcasse plus importante. C'est le cas des régions rachidienne, sous-lombaire, et crurale postérieure. 
TABLEAU 3

Moyennes $(\bar{x})$, coefficients de variation $(v)$, coefficients de variation résiduels $\left(v_{r}\right)$ à poids de tissu musculaire constant, corrélations partielles $(r)$ avec le poids total du tissu musculaire, à poids de carcasse constant, pour les poids des régions musculaires mesurés en grammes, obtenues par dissection de la partie droite de la carcasse.

\begin{tabular}{|c|c|c|c|c|c|c|c|c|c|c|c|c|c|}
\hline \multirow{2}{*}{\multicolumn{2}{|c|}{ Caractères }} & \multicolumn{4}{|c|}{ Souche $A$} & \multicolumn{4}{|c|}{ Souclue $B$} & \multicolumn{4}{|c|}{ Souche $C$} \\
\hline & & $\bar{x}$ & $v$ & $v_{r}$ & $r$ & $\bar{x}$ & $v$ & $v_{r}$ & $r$ & $\vec{x}$ & $v$ & $v_{r}$ & $r$ \\
\hline$X$ I I & $\begin{array}{l}\text { Région ra }- \\
\text { chidienne ... }\end{array}$ & 87,0 & $2 \mathrm{I}, 4$ & 6,0 & 0,55 & 106,0 & $\mathbf{1 6 , 6}$ & 5,5 & 0,53 & 80,6 & $\mathrm{r} 8,5$ & $7, \mathbf{I}$ & 0,36 \\
\hline$X 12$ & $\begin{array}{l}\text { Région ven- } \\
\text { trale } \ldots \ldots \ldots\end{array}$ & 3,7 & 25,6 & $2 \mathrm{I}, 9$ & $0,2 \mathrm{I}$ & 4,5 & 27,3 & $23, I$ & 0,35 & 4,1 & 29,8 & 27,7 & 0,08 \\
\hline$X$ ז 3 & $\begin{array}{l}\text { Région cer- } \\
\text { vicale, ven- } \\
\text { trale et dor- }\end{array}$ & & & & & & & & & & & & \\
\hline$X_{14}$ & $\begin{array}{l}\text { sale } \ldots \ldots \ldots \\
\text { Région dor- }\end{array}$ & $23, I$ & 24,5 & 18,0 & 0,06 & 25,8 & $\mathrm{I} 9, \mathrm{I}$ & $\mathbf{I} 2,3$ & 0,08 & 20,6 & 20,7 & $\mathrm{I} 4,2$ & O,OI \\
\hline 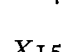 & so-spinale .. & 5,7 & 31,9 & 23,7 & $0, I_{4}$ & $6, \mathbf{I}$ & 25,9 & 22,8 & 0,00 & 5,3 & $33, \mathrm{I}$ & 24,0 & 0,23 \\
\hline$x+6$ & tale ........ & 24,4 & 20,7 & I 5,7 & $-0, \mathrm{I} \mathrm{I}$ & 26,4 & $\mathrm{I} 8,5$ & I 2,6 & $0, \mathrm{x} 3$ & 23,4 & 20,4 & $\mathrm{I} 7, \mathrm{I}$ & 0,07 \\
\hline$X 16$ & $\begin{array}{l}\text { Région pec- } \\
\text { torale ....... }\end{array}$ & 12,8 & $3 I, 3$ & 20,5 & $0,2 \mathrm{I}$ & I 4,6 & 20,9 & I 6,4 & 0,09 & I 1,6 & 23,2 & $\mathrm{I} 8, \mathrm{I}$ & 0,23 \\
\hline $\begin{array}{l}X I 7 \\
X I 8\end{array}$ & $\begin{array}{l}\text { Diaphragme. } \\
\text { Région abdo- } \\
\text { minale infé- }\end{array}$ & 5,5 & I 9,0 & 10,2 & 0,18 & 6,0 & I 7,0 & $\mathrm{I} 2,3$ & $\mathrm{O}, \mathrm{I} 7$ & 5,3 & $\mathrm{I} 8, \mathrm{I}$ & I I, I & 0,30 \\
\hline$X 19$ & $\begin{array}{l}\text { rieure } \ldots . . . \\
\text { Région sous- }\end{array}$ & 34,3 & 20,2 & 10,2 & $-0,07$ & 40,7 & I 6,4 & 9,9 & $\mathrm{O}, \mathrm{I} 5$ & 35,0 & I 8,6 & 9,3 & $0, \mathbf{1} 8$ \\
\hline & lombaire ... & I9,2 & $20, \mathrm{I}$ & I3,I & 0,44 & 24,3 & I 8,6 & 8,2 & 0,27 & I $9, \mathbf{I}$ & $2 \mathrm{I}, 4$ & I I , I & 0,30 \\
\hline$X 20$ & Épaule ..... & I 6,5 & I6,7 & 8,9 & $0,3 \mathbf{I}$ & I 8,7 & 14,6 & 6,6 & 0,45 & 14,2 & I 8,9 & 9,5 & 0,43 \\
\hline$X 2 \mathrm{I}$ & Bras $\ldots \ldots$ & $\mathrm{I} 4, \mathrm{I}$ & I 8,5 & 8,2 & 0,43 & I6,0 & $\mathrm{I} 5, \mathrm{I}$ & 6,9 & 0,36 & I 2,6 & I 7,2 & $8, \mathrm{I}$ & 0,10 \\
\hline $\begin{array}{l}X 22 \\
X 23\end{array}$ & $\begin{array}{l}\text { Avant-bras } \\
\text { Région fes- }\end{array}$ & 5,5 & 20,8 & 12,3 & $0,5 \mathrm{I}$ & 6,2 & $\mathrm{I} 7,2$ & I I ,2 & 0,25 & 5,0 & I 8,3 & 9,6 & o, I 7 \\
\hline$X_{24}$ & $\begin{array}{l}\text { sière ...... } \\
\text { Région cru- } \\
\text { rale posté- }\end{array}$ & I 3,I & 22,5 & 10,6 & 0,28 & I 5,2 & $\mathrm{I} 7,2$ & I0,6 & 0,17 & I I, I & 20,2 & I 5,9 & $-0,09$ \\
\hline$X 25$ & $\begin{array}{l}\text { rieure ...... } \\
\text { Région cru- } \\
\text { rale antéri- }\end{array}$ & 45,6 & 20,8 & 6,7 & 0,46 & 56,5 & 13,6 & 4,2 & 0,56 & 42,3 & I 7,4 & 9,3 &, 17 \\
\hline$X_{26}$ & $\begin{array}{l}\text { eure } \ldots \ldots \\
\text { Région cru- }\end{array}$ & 30,4 & 18,7 & 7,8 & $0,3^{\circ}$ & 36,2 & 14,9 & 7,0 & $-0, O I$ & 27,2 & I6,0 & 7,6 & 0,09 \\
\hline$X 27$ & $\begin{array}{l}\text { rale interne. } \\
\text { Région pelvi- }\end{array}$ & I 6,5 & 20,5 & 12,0 & 0,30 & I9,5 & 16,8 & 7,5 & 0,09 & I6,4 & 25,0 & 20,5 & 0,04 \\
\hline & & 8,0 & 34,2 & 32,2 & 0,03 & 8,9 & 29,5 & $27, I$ & 0,35 & 6,9 & 27,8 & 24,6 & 0,27 \\
\hline & jambe..... & I 9,0 & 19,3 & 7,7 & $0,4^{8}$ & 23,9 & I 5,2 & 6,3 & $0, \mathbf{I} 3$ & I 8,2 & I 8,4 & I I ,3 & 0,10 \\
\hline
\end{tabular}

En ce qui concerne les os du squelette étudiés, on ne trouve pas comme pour les régions musculaires, que la variabilité de leur répartition dans l'ensemble du tissu osseux est systématiquement plus élevée que celle de celui-ci dans la carcasse. Seuls quelques os particuliers présentent une grande variabilité résiduelle. Pour certains d'entre eux, par exemple pour les os des mains $\left(v_{r}=\mathrm{r}_{4}, 2 \%\right.$ à

Annales de Génétique et de Sélection animale. - r97o. 
TABLEAU 4

Moyennes $(\bar{x})$, coefficients de variation $(v)$, coefficients de variation résiduels $\left(v_{r}\right)$, à poids de squelette constant, corrélations partielles $(r)$ avec le poids total du tissu osseux, à poids de carcasse constant, pour les poids des os obtenus par dissection, mesurés en grammes.

\begin{tabular}{|c|c|c|c|c|c|c|c|c|c|c|c|c|c|}
\hline & Caractères & $\bar{x}$ & $v$ & $v_{r}$ & $r$ & $\bar{x}$ & $v$ & $v_{r}$ & $r$ & $\bar{x}$ & $v$ & $v_{r}$ & $r$ \\
\hline$X 29$ & Bassin & $\mathrm{I} \mathrm{I}, 7$ & $13, \mathrm{I}$ & 5,8 & 0,76 & I 3,7 & $\Upsilon 2,7$ & 6,3 & 0,68 & I I , 5 & I 5,7 & 9,5 & 0,66 \\
\hline$X_{30}$ & Fémurs & 19,3 & II, 6 & 5,8 & 0,69 & 22,4 & 13,0 & 6,9 & 0,56 & 18,5 & $\begin{array}{r}15,5 \\
\end{array}$ & 7,9 & 0,66 \\
\hline$X_{31}$ & Tibias et péronés & I 5,5 & 14,0 & 7,0 & 0,69 & 17,6 & I 3,0 & 6,9 & 0,60 & 15,0 & 15,9 & 9,6 & 0,58 \\
\hline$X_{32}$ & Os des pieds et & & & & & & & & & & & & \\
\hline$X 33$ & $\begin{array}{l}\text { tarses } \ldots \ldots \ldots \ldots \\
\text { Omoplates et cia- }\end{array}$ & $\mathrm{I} 8, \mathrm{I}$ & $16, \mathbf{I}$ & I I, 7 & 0,46 & 20,0 & I5,7 & 9,5 & $0,6 \mathbf{I}$ & $\mathrm{I} 7,2$ & 16,4 & I I , 5 & $0,4^{8}$ \\
\hline & vicules ........ & 5,2 & 20,7 & 16,7 & 0,34 & 5,4 & 14,7 & $8, \mathbf{I}$ & $0,64 \|$ & 5,0 & 20,9 & I3,9 & $0,5^{\circ}$ \\
\hline$X_{34}$ & Huméru & $9, \mathrm{I}$ & 14,0 & 7,5 & 0,64 & $\mathrm{IO}, \mathrm{I}$ & 13,9 & 7,2 & 0,60 & 8,5 & 15,0 & 8,0 & 0,62 \\
\hline $\begin{array}{l}X_{35} \\
X_{36}\end{array}$ & $\begin{array}{l}\text { Radius et cubitus } \\
\text { Os des mains et }\end{array}$ & 7,6 & 16,2 & 9,4 & 0,69 & 8,3 & $\mathrm{I} 4, \mathrm{I}$ & 9,3 & 0,43 & $7, \mathrm{I}$ & $\mathbf{1} 8,9$ & $I I, 2$ & 0,68 \\
\hline & carpe & 5,8 & 22,2 & 17,9 & 0,52 & 6,3 & 19,7 & 14,2 & 0,38 & $6, \mathrm{I}$ & $\mathbf{I} 7,3$ & 15,2 & 0,27 \\
\hline$X_{37}$ & Sternum et côtes & I0,6 & 14,7 & 9,8 & 0,55 & $\mathrm{I} 2,3$ & 13,5 & 7,8 & 0,58 & I I, 4 & 25,5 & $20, \mathrm{I}$ & 0,32 \\
\hline & brale ........... & 38,2 & $2 \mathrm{I}, 7$ & $\mathbf{1 4}, 6$ & 0,60 & 45,0 & $\mathbf{r} 4,4$ & 8,6 & 0,66 & 40,6 & I9,3 & I I ,9 & 0,73 \\
\hline$X 39$ & Os de la têt & 39,5 & I 7,0 & I0,9 & 0,74 & 43,6 & $\mathrm{x} 7,4$ & 13,0 & 0,69 & 40,6 & $r_{4,9}$ & I 2,4 & 0,45 \\
\hline
\end{tabular}

I7,9\%), cela peut correspondre à la difficulté de leur dissection. Les coefficients de variation trouvés sont dans l'ensemble légèrement plus élevés que ceux cités par Latimer et SAwin (I962) concernant des poids d'os secs de 35 mâles adultes de leur race III. Tous les os considérés permettent d'améliorer de façon non négligeable la précision de l'estimation du poids total du squelette après prise en compte du poids de carcasse. On pourrait donc envisager dans ce but une dissection très simplifiée permettant d'obtenir les poids des os de la tête, des pieds et mains, des membres.

\section{5. - Variabilité génétique intra-souche}

\section{I. - Valeurs brutes des caractères}

Pour tous les caractères considérés, la composante mère de la variance, significativement supérieure à o dans tous les cas, est très supérieure à la composante père, sauf pour les poids du tractus digestif où elle est du même ordre de grandeur. Cela justifie de calculer l'estimation de l'héritabilité des caractères à partir de la composante père de la variance et non à partir de la moyenne des deux composantes père et mère. La différence entre les deux composantes pourrait être due à la présence d'effets maternels, de variances génétiques liées au sexe et de dominance ou d'épistasie.

La composante père de la variance du poids vif conduit à une estimation relativement faible de l'héritabilité $\left(h^{2}=0 ; \mathbf{I} 3\right)$. La composante mère est de l'crdre 
TABLEAU 5

Fractions de la variance phénotypique, en p. Ioo, dues à la variance des effets race (4), père (3), mère (2), intra-mère (1), et composante père de l'héritabilité $\left(h^{2}\right)$ intra-race

\begin{tabular}{|c|c|c|c|c|c|}
\hline Caractères & (I) & (2) & (3) & (4) & $h^{2}$ \\
\hline 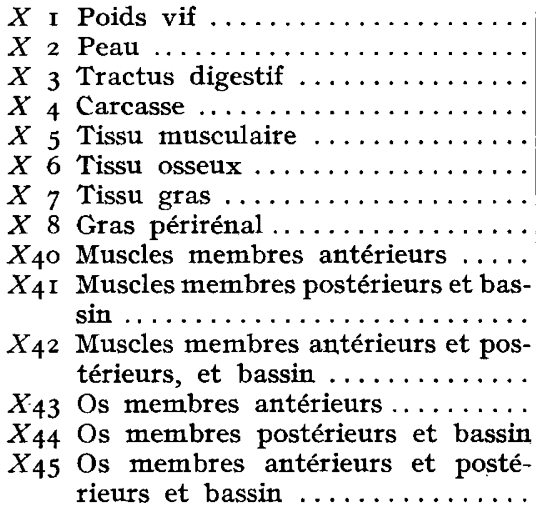 & $\begin{array}{l}29,4 \\
38,7 \\
33,9 \\
28,6 \\
30,0 \\
43,5 \\
40,8 \\
37,6 \\
30,0 \\
27,1 \\
27,1 \\
54,8 \\
32,8 \\
35,3\end{array}$ & $\begin{array}{l}37,2 \\
48,8 \\
30,0 \\
35,4 \\
32,3 \\
32,8 \\
39,7 \\
37,9 \\
29,5 \\
28,0 \\
28,5 \\
29,7 \\
31,3 \\
31,7\end{array}$ & $\begin{array}{c}2,3 \\
* \\
21,0 \\
1,8 \\
3,4 \\
* \\
3,4 \\
3,5 \\
5,7 \\
3, \mathbf{1} \\
\\
3,7 \\
7,9 \\
6,8 \\
\\
8,5\end{array}$ & $\begin{array}{r}3 \mathrm{I}, \mathrm{I} \\
\mathrm{I} 4, \mathrm{I} \\
\mathrm{I} 5, \mathrm{I} \\
34,2 \\
34,2 \\
23,8 \\
\mathrm{I} 6, \mathrm{I} \\
2 \mathrm{I}, \mathrm{O} \\
34,8 \\
4 \mathrm{I}, 7 \\
40,7 \\
7,7 \\
29, \mathrm{I} \\
24,4\end{array}$ & $\begin{array}{c}0, \mathrm{I} 3 \\
* \\
0,99 \\
0, \mathrm{I} I \\
0,2 \mathrm{I} \\
* \\
0, \mathrm{I} 6 \\
0, \mathrm{I} 8 \\
0,35 \\
0,21 \\
0,25 \\
0,34 \\
0,38 \\
0,45\end{array}$ \\
\hline
\end{tabular}

(*) Estimation négative ou nulle de la composante de la variance.

de 5 fois plus grande. VENGE (1953) ne met pas nettement en évidence un effet maternel (cytoplasmique ou du milieu) persistant après 1'âge de 56 jours, sur le poids corporel du lapin. JoHANSSON et VENGE (I953) trouvent une influence de la taille de la portée à la naissance sur les poids individuels, qui est maximale à l'âge de 3 semaines, mais qui persiste encore à l'âge de II 7 jours où elle représente, pour certains groupes d'animaux, de l'ordre de II p. Ioo de la variance, alors qu'elle en représente en moyenne 23,4 p. Ioo à l'âge de 56 jours. Sur un échantillon d'effectif plus important (résultats non publiés) d'animaux contemporains élevés dans les mêmes conditions que ceux disséqués et étudiés actuellement, le nombre de nés vivants par portée présente une corrélation moyenne, suivant les trois races et les deux sexes, de $-0,44$ avec le poids individuel à 84 jours, ce qui rend donc compte de I9 p. Ioo de la variance de ces poids. L'effet taille de portée a donc pu augmenter la valeur de la composante mère de la variance. Il a pu en être de même d'un effet numéro de portée, ainsi que d'un effet milieu commun, jusqu'au sevrage, aux individus d'une même portée. De plus, le fait que les portées n'aient pas été toutes abattues exactement au même âge, a pu augmenter la valeur de la composante mère de la variance. YAO et EATON (I954) trouvent un effet d'hétérosis significatif pour le poids d'abattage (à r8o j) de lapins Néo-Zélandais blancs, issus de croisement de lignées consanguines. Il est donc possible que la variance des effets mère comprenne une part de variance génétique de dominance et d'épistasie. Par ailleurs, les lapins étudiés ici étaient des mâles. Dans le cas de gènes liés au sexe, la composante mère de la variance pour les descendants du sexe mâle 
est plus grande que la composante père de la variance, et inversement chez les descendants femelles. BEILHARZ (I950), analysant les poids corporels de lapins mâles et femelles âgés de 4 semaines, trouve des résultats pour les valeurs des composantes père et mère des deux sexes qui laissent supposer l'existence d'une hérédité liée au sexe. Il est donc possible que dans le cas présent la composante mère de la variance comprenne une part de variance génétique liée au sexe.

Pour les poids de carcasse, du tissu musculaire, du tissu gras, les héritabilités sont du même ordre de grandeur que pour le poids vif. Cela s'explique du fait que les corrélations génétiques mère et père sont fortes entre le poids vif d'une part et d'autre part le poids de carcasse $(r=0,996$ et $r=0,868)$, le tissu musculaire $(r=0,966$ et $0,9 \mathrm{I} 4)$, le tissu gras $(r=0,92 \mathrm{I}$ et $r=-\mathrm{I}, \mathrm{I05})$. Curieusement, la corrélation génétique père entre les poids vif et du tissu gras est négative, ce qui semble correspondre à une particularité de 1'échantillon étudié.

Trois héritabilités s'écartent nettement des valeurs trouvées pour la plupart des caractères : celle du poids tractus digestif qui est très élevée, celles des poids de la peau et du squelette total dont les estimations sont négatives et très voisines de $o$. En ce qui concerne le tissu osseux, la composante père de la variance est négative et très faible pour son poids total, alors que pour le poids des os des membres antérieurs, postérieurs et du bassin (variables $X_{43}, X_{44}, X_{45}$ ), elle conduit à des valeurs des héritabilités, de l'ordre de 0,34 à 0,45 , plus élevées que pour les autres caractères. Cela montre l'intérêt de considérer le poids de ces os. D'une part, ils sont de dissection facile et les erreurs de mesure sont faibles, d'autre part, ils semblent être relativement moins sensibles aux variations du milieu que les autres caractères considérés.

5.2. - Répartition de la peau, du tube digestif, du poids de carcasse, par rapport au poids vif

TABLEAU 6

Fractions de la variance phénotypique intra-souche, en p. ıoo, dues à la variance des effets père (3), mève (2), intra-mère (I), composante père de l'héritabilité $\left(h^{2}\right)$, pour les variables écarts

\begin{tabular}{|c|c|c|c|c|}
\hline Variables & (I) & (2) & (3) & $h^{2}$ \\
\hline 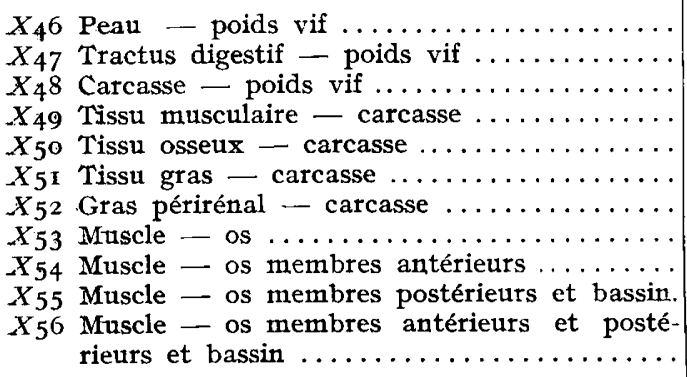 & $\begin{array}{l}59,7 \\
41,3 \\
65,9 \\
64,1 \\
85,7 \\
56,6 \\
61,4 \\
58,4 \\
54,2 \\
63,6 \\
63,8\end{array}$ & $\begin{array}{r}36,2 \\
32, I \\
11,4 \\
36,8 \\
8, I \\
19,4 \\
25,4 \\
31,7 \\
40,3 \\
25,9 \\
27, I\end{array}$ & $\begin{array}{r}4,2 \\
26,7 \\
22,7 \\
6 \\
6,2 \\
28,8 \\
13,2 \\
9,8 \\
5,5 \\
10,4 \\
9,2\end{array}$ & $\begin{array}{c}0,17 \\
1,07 \\
0,91 \\
* \\
0,25 \\
1,15 \\
0,53 \\
0,39 \\
0,22 \\
0,42 \\
0,37\end{array}$ \\
\hline
\end{tabular}

$\left.{ }^{*}\right)$ Estimation négative ou nulle de la composante de la variance. 
Il apparaît un déterminisme génétique additif important pour les poids du tractus digestif et de carcasse, à poids vif constant. Le poids de carcasse à poids vif constant est un critère zootechnique important à considérer car il correspond à une autre expression du rendement à l'abattage. L'estimation de son héritabilité, en faisant la moyenne des composantes père et mère est $h^{2}=0,68$. La composante père de l'héritabilité est égale à $0,9 \mathrm{I}$. Bien que 1'intervalle de confiance de ces estimations soit grand, on peut penser que l'héritabilité de cette variable écart poids de carcasse-poids vif est moyenne à élevée, dans les conditions standardjsées d'élevage. Nous ne connaissons pas de références donnant des valeurs de l'estimation de l'héritabilité du rendement à l'abattage chez le Lapin. Celle trouvée ici est du même ordre de grandeur que celle indiquée par RICARD (I965) chez le poulet $\left(h^{2}=0,40\right)$, et que celle citée par Christians et al. (I962) chez les bovins Angus $\left(h^{2}=0,74\right)$ élevés en " feed-lot».

\section{3. - Répartition des trois principaux tissus par rapport au poids de carcasse}

Nous avons ici considéré les poids de chacun des tissus exprimés en écarts des valeurs observées aux valeurs estimées par leurs droites de régression (dont la pente est calculée intra-famille) sur le poids de carcasse. La composante père de l'héritabilité de l'écart poids du tissu musculaire - poids de carcasse est pratiquement nulle, celle de l'écart poids de tissu osseux - poids de carcasse, est un peu plus élevée. L'héritabilité de l'écart poids de tissu gras - poids de carcasse est très élevée. Il serait donc aisé de sélectionner pour obtenir le pourcentage de gras désiré dans les carcasses de lapins élevés dans des conditions homogènes d'alimentation. Nous avons trouvé un résultat comparable chez le Poulet (RICARD et Rouvies, r969) où l'héritabilité du poids de gras périrénal à poids de carcasse constant, chez des animaux de même âge, est moyenne à élevée. Chez le Lapin, dans une dissection simplifiée, le poids total de gras pourrait être remplacé par celui de gras périrénal dont l'héritabilité à poids de carcasse constant, ici trouvée, est de 0,53 , la corrélation génétique de ces deux caractères étant égale à 0,95 .

\section{4. - Écart muscle-os}

Les écarts des valeurs observées du poids total des muscles à leurs valeurs estimées par leur droite de régression, calculée intra-famille, sur le poids total d'os, présentent une héritabilité de 0,39 . Cela confirme l'intérêt de considérer ce critère de sélection pour modifier la composition anatomique, sa variabilité génétique étant plus grande que celle des écarts du tissu musculaire ou du tissu osseux, par rapport au poids de carcasse. Nous avons fait la même constatation chez le Poulet (Rouvier et RICARD, I967b). L'écart muscle-os étant en corrélation positive avec le poids de carcasse, nous avons calculé son héritabilité après correction pour les différences de poids de carcasse. Intra-souche, la corrélation entre phénotypes de deux demi-frères est, pour ce dernier critère, de 0,046 et celle entre phénotypes de pleins frères est de 0,206 . Il semble donc subsister une variation génétique de l'écart muscle-os, chez des animaux de même âge et même poids de carcasse.

Les héritabilités des écarts muscle-os dans certaines régions corporelles 
(membres antérieurs, membres postérieurs et bassin) sont du même ordre de grandeur que celles trouvées pour cet écart dans l'ensemble de la carcasse. Les corrélations génétiques, tenant compte des composantes mère et père des variances et covariances, entre les écarts muscle-os des trois régions considérées et l'écart muscle-os dans la carcasse, varient de $0,9 \mathrm{I}$ à 0,95 . On pourrait donc envisager une sélection indirecte efficace à partir d'une dissection simplifiée. La technique qui conduirait aux progrès les plus rapides serait celle basée sur la dissection des deux membres postérieurs et du bassin.

\section{6. - Variabilité entre souches}

\section{TABLEAU 7}

Corrélations et régressions, signification du test de comparaison aes moyennes ajustées (analyse de covariance) : NS, non significatif - S, significatif - TS, très significatif

\begin{tabular}{|c|c|c|c|c|c|c|c|c|}
\hline $\begin{array}{l}\text { Variable } \\
\text { indépen- } \\
\text { dante }\end{array}$ & Unité & $\begin{array}{l}\text { Variable } \\
\text { dépendante }\end{array}$ & Unité & Race & $\begin{array}{l}\text { Corré- } \\
\text { lation }\end{array}$ & $\begin{array}{l}\text { Régres- } \\
\text { sion }\end{array}$ & $\begin{array}{l}\text { Régression } \\
\text { commune }\end{array}$ & $\begin{array}{c}\text { Test de } \\
\text { compa- } \\
\text { raison } \\
\text { des } \\
\text { moyennes } \\
\text { ajustées }\end{array}$ \\
\hline Poids vif & $g$ & $\begin{array}{l}\text { Poids } \\
\text { de carcasse }\end{array}$ & $\mathrm{g}$ & $\begin{array}{l}A \\
B \\
C\end{array}$ & $\begin{array}{l}0,984 \\
0,984 \\
0,981\end{array}$ & $\begin{array}{l}0,694 \\
0,663 \\
0,662\end{array}$ & $0,675 \pm 0,007$ & TS \\
\hline $\begin{array}{c}\text { Poids } \\
\text { de carcasse }\end{array}$ & $\mathrm{g}$ & $\begin{array}{l}\text { Poids } \\
\text { du tissu } \\
\text { musculaire }\end{array}$ & $\mathrm{g}$ & $\begin{array}{l}A \\
B \\
C\end{array}$ & $\begin{array}{l}0,976 \\
0,981 \\
0,975\end{array}$ & $\begin{array}{l}0,649 \\
0,630 \\
0,645\end{array}$ & $0,641 \pm 0,022$ & NS \\
\hline $\begin{array}{c}\text { Poids } \\
\text { de carcasse }\end{array}$ & $\mathrm{g}$ & $\begin{array}{l}\text { Poids } \\
\text { du tissu } \\
\text { osseux }\end{array}$ & $\mathrm{g}$ & $\begin{array}{l}A \\
B \\
C\end{array}$ & $\begin{array}{l}0,734 \\
0,785 \\
0,768\end{array}$ & $\begin{array}{l}0,080 \\
0,091 \\
0,094\end{array}$ & $0,088 \pm 0,012$ & Ts \\
\hline $\begin{array}{c}\text { Poids } \\
\text { de } \text { carcasse }\end{array}$ & $\mathrm{g}$ & $\begin{array}{l}\text { Poids } \\
\text { du tissu gras }\end{array}$ & $\mathrm{g}$ & $\begin{array}{l}A \\
B \\
C\end{array}$ & $\begin{array}{l}0,807 \\
0,807 \\
0,826\end{array}$ & $\begin{array}{l}0,139 \\
0,141 \\
0,129\end{array}$ & $0, \mathrm{I}_{34} \pm \mathrm{o}, \mathrm{O}$ I 5 & $\mathrm{~s}$ \\
\hline $\begin{array}{c}\text { Poids } \\
\text { de carcasse }\end{array}$ & $\mathrm{kg}$ & $\begin{array}{l}\text { Rapport } \\
\text { muscle /os }\end{array}$ & pr & $\begin{array}{l}A \\
B \\
C\end{array}$ & $\begin{array}{l}0,626 \\
0,488 \\
0,491\end{array}$ & $\begin{array}{l}0,174 \\
0,103 \\
0,134\end{array}$ & $0,139 \pm 0,034$ & NS \\
\hline $\begin{array}{c}\text { Poids } \\
\text { du squelette }\end{array}$ & $\mathrm{g}$ & $\begin{array}{l}\text { Poids } \\
\text { du tissu } \\
\text { musculaire }\end{array}$ & $\mathrm{g}$ & $\begin{array}{l}A \\
B \\
C\end{array}$ & $\begin{array}{l}0,67 \mathrm{I} \\
0,744 \\
0,659\end{array}$ & $\begin{array}{l}4,073 \\
4,115 \\
3,55^{2}\end{array}$ & $3,955 \pm 0,642$ & TS \\
\hline
\end{tabular}

Il semble intéressant de comparer les échantillons des trois souches bien que les conclusions ne puissent pas s'appliquer, de façon générale, aux trois races dont elles sont issues.

Le rendement à l'abattage est plus élevé dans la souche $\mathrm{B}$. On observe de même des différences entre les trois souches pour les rapports des poids des trois 
tissus au poids de carcasse chaude : respectivement pour chacune des trois souches, les pourcentages moyens concernant le tissu musculaire obtenu par dissection sont de 57,$3 ; 58,6 ; 56,9$; ceux concernant le squelette sont de I3,3; I2,9; I4,2; ceux concernant le tissu adipeux sont de 6,$3 ; 6,7 ; 5,7$. Les animaux de la souche $B$ ont, en plus d'un meilleur rendement à l'abattage, un meilleur pourcentage de muscles dans la carcasse et un rapport muscle/os significativement supérieur à celui des autres races. Cependant, une partie de ces différences sont liées aux différences de poids vif, donc de vitesse de croissance, et de poids de carcasse.

L'analyse de covariance indique qu'à poids vif constant, il existe des différences très significatives entre les poids moyens de carcasse des animaux des trois souches. Celles-ci se rangent dans 1'ordre $B, A, C$. Cependant la différence entre $B$ et $A$ n'est pas significative. La différence qui existe est donc entre $B$ et $A$ d'une part, $C$ d'autre part.

Il n'y a pas de différence significative entre souches, du poids du tissu musculaire à poids de carcasse constant. Par contre, il y a des différences pour les poids du squelette et du tissu adipeux, la souche $A$ ayant le squelette le plus léger et le plus de gras, lorsqu'on ramène les trois souches au même poids de carcasse. Si l'écart muscle-os varie significativement entre souches, il ne présente plus de variation significative lorsque celles-ci sont ramenées au même poids de carcasse. Les caractères qui présentent une variation significative entre souches sont les mêmes que ceux présentant une variation génétique intra-souche, à l'exception de l'écart muscle-os à poids de carcasse constant.

Les pentes des droites de régression données dans le tableau 7 permettraient d'estimer, dans l'intervalle de poids vif considéré ( $1600 \mathrm{~g}$ à $3000 \mathrm{~g}$, approximativement), pour des groupes d'animaux mâles de 3 races de format adulte comparable, le poids moyen de carcasse chaude connaissant le poid vif moyen. Elles permettent également d'estimer les poids des trois tissus osseux, musculaire et gras, en fonction du poids de carcasse, et donc de suivre l'évolution de la composition anatomique des carcasses, en fonction de leur poids. Cette estimation est plus précise, pour un même nombre d'animaux contrôlés, pour les poids de carcasse et des muscles totaux, que pour ceux des tissus osseux et gras.

\section{CONCLUSION}

L'étude ne porte que sur un échantillon d'animaux d'effectif limité, ce qui a été rendu nécessaire du fait d'une dissection très complète des carcasses. Il s'en dégage cependant des premières conclusions, aussi bien dans le domaine biologique que pratique.

Sur les plans de l'analyse statistique et de l'interprétation biologique, nous avons préféré exprimer l'importance d'un caractère relativement à un autre par l'écart de la valeur observée à celle estimée par la droite de régression de l'un sur l'autre. Nous avons ainsi obtenu des variąbles " écarts".

Au point de vue biologique, les résultats obtenus s'expliquent, compte tenu des lois moyennes de l'allométrie de croissance chez le Lapin et de variations individuelles, en grande partie d'origine génétique, autour de ces lois moyennes. 
Sur le plan pratique, les résultats fournissent des indications sur le choix des meilleurs critères de sélection pour modifier le rendement à l'abattage indépendamment de la vitesse de croissance, et la composition anatomique des carcasses. L'héritabilité de la variable écart poids de carcasse-poids vif, est élevée. Il s'ensuit qu'on peut espérer réaliser des progrès rapides pour ce caractère, en sélectionnant sur descendance ou sur collatéraux, en maintenant constants l'âge et le poids vif d'abattage. Nous avons récemment établi chez le poulet (RICARD et Rouvier, I970) des index de sélection suivant ce principe, et étudié leurs efficacités. Il serait également possible, la vitesse de croissance restant constante, de diminuer l'âge d'abattage permettant d'obtenir un poids de carcasse donné.

La variabilité génétique additive de la composition anatomique s'exprime au mieux par l'écart muscle-os et une variable donnant le poids du tissu gras à poids de carcasse constant. Compte tenu de 1'héritabilité de l'écart muscle-os, on peut envisager aussi des progrès rapides en sélectionnant pour augmenter le poids du tissu musculaire tout en gardant le poids du squelette constant. Une sélection éventuelle pour modifier la composition anatomique pourrait s'effectuer à partir des résultats d'une dissection simplifiée donnant les poids de muscle et d'os dans les membres postérieurs et le bassin où à défaut dans les deux membres antérieurs, ainsi que le poids de gras périrénal.

Les variables relatives à un bon rendement à 1'abattage et à une bonne composition anatomique, varient dans le même sens. Il conviendrait cependant, dans une sélection, compte-tenu des objectifs recherchés, de préciser l'importance que l'on attribue à chacune d'elles.

L'existence de différences entre souches, en plus des différences génétiques intra-souche, indique l'intérêt d'effectuer leur comparaison avant de commencer la sélection intra-souche sur les meilleures d'entre elles. Ces problèmes de sélection sur le rendement à l'abattage et la composition anatomique devraient cependant être discutés dans le cadre plus général de l'amélioration de l'élevage des lapereaux entre le sevrage et l'abattage, d'autres caractères telle que la vitesse de croissance et l'indice de consommation dans cette période étant à considérer.

Rę̧u pour publication en mai 1970.

\section{REMERCIEMENTS}

Nous tenons à remercier MM. J. Cantier et L. Ollivier qui nous ont apporté leurs critiques constructives lors de la lecture de notre manuscrit.

\section{SUMMARY}

\section{GENETIC VARIABILITY OF SIAUGHTER YIELD AND ANATOMICAL, COMPOSITION IN THREE BREEDS OF RABBITS}

160 male rabbits from three breeds produced under a hierarchical mating system (breed. father-mother-offspring), were killed at $84 \pm 2$ days of age. The present study involves: live weight at slaughter, skin weight and weight of digestive tract emptied of its contents, hot carcass weight, total weight of muscle, bone and fatty tissues and of areas or parts of these 
tissues, muscle /bone ratio (muscle weight /bone weight), dressing percentage, differences between the observed values of a character and their values calculated by its regression line on another.

The statistical parameters of the variables studied are given for each one of the three breeds (table 1). Live and carcass weight, muscular tissue and skin are moderately variable (coefficients of variation $\mathrm{V}=12$ to $18 \mathrm{p}$. 100). The weight of the digestive tract and skeleton vary little, while that of fatty tissue is very variable. At constant live weight, carcass weight variability is low, while that of the skin and digestive tract are higher. At constant carcass weight, muscular tissue varies little $(V=2.8$ to 4 p. 100), while fatty tissue is very variable $(V=20$ to 25 p. 100). Muscle/bone ratio or muscular tissue weight at constant skeleton weight remains moderately variable.

Variability of distribution (table 3) of areas of muscular tissue in relation to the latter is higher than that of muscular tissue at constant carcass weight. Only a small number of muscle area weights can be used to obtain a good estimation of total muscular weight by using a multiple linear equation involving carcass weight plus muscle-area weight. All the bones under consideration furnish significant complementary information of the same order (table 4$)$ for estimating bone tissue weight in a regression line equation in which carcass weight is a factor.

Heritability estimates (table 5) of raw values of skin and bone tissue weight are not signifiant. Those of live weight and carcass weight are low. Some hypothesis may explain the high values of the dam variance component found in that case. For other characters, heritability estimated are average to high.

Additive genetic variability of distribution of body parts (skin, digestive tract, carcass) in relation to live weight is large.

The same is true of variables expressing the relative importance of muscular tissue in relation to the skeleton, or of fatty tissue in the carcass.

Results show that an efficient selection for increasing slaughter yield is possible, even at constant age and live slaughter weight. Also, it even seems possible at constant age and carcass weight to make rapid progress using selection for modifying anatomical composition. This could be accomplished by increasing the relative importance of muscular tissue in relation to the skeleton. This may be done using the results of a simplified dissection giving the tissue weights of certain carcass areas.

\section{RÉFÉRENCES BIBLIOGRAPHIQUES}

Bemlharz R. G., I960. Research into sex linked control of body weight in poultry and Rabbits. Proc. A ust. Soc. Anim. Prod., 3, r39-146.

BERg R. T., BuTterfield R. M., I966. Muscle : Bone ratio and fat percentage as measures of beef carcass composition. Anim. Prod., 8, I-II.

CANTIER J., VezINHet A., r968. Guide pour la dissection et l'identification des principaux muscles de la carcasse chez le lapin. Ann. Biol. anim. Bioch. Biophys., 8, ro7-r 39.

Cantier J., Vezinhet A., Rouvier R., Dauzier L., 1969. Allométrie de croissance chez le Lapin. I. Principaux organes et tissus. Ann. Biol. anim. Bioch. Biophys., 9, 5-39.

Christians C. J., Chambers D., Walters I. E., Whiteman J. V., Stephens D. F., I962. Heritability estimates of beef characteristics. J. Anim. Sci., 21, $3^{87}$.

Damjanova $\mathrm{N}$., I966. The growth and fattening characters of Vienchin rabbits in relation to age and sex. Nauchni Trud. vissh selskostop. Inst. Georgi Dimitrov Zootekh. Fak., 16, 469 (in Anim. Breed. Abstr., 35: $401 \mathrm{I}$ ).

Dinkel C. A., Wilson I. L., Tuma H. J., Minyard J. A., 1965. Ratios and percents as measures of carcass traits. J. anim. Sci., 24, 425-429.

Dumont B. I.., LE Guelte P., ARnoux J., I96r. Étude biométrique des bovins de boucherie. I. Variabilité de la composition anatomique de la carcasse des bovins charolais. Ann. Zootech., 10, I 49 -154.

FraCANZANI, 1966. The production of rabbits for meat. Riv. Zootech., 39, 222-226.

Johansson I., Venge O., 1953. A study of the variation in weight during growth, and in some skeletal characteristics of adult rabbits. Ann. R. Agric. coll. Sweden, 19, I6I-I95.

JADWIGA K., I963. Investigations on the fur quality and slaughter value of rabbits of the Vienna white breed. Roczn. Nauk. rol. B., 82, I69-181 (in Anim. Breed. Abstr., 33; r646).

LATIMER H. B., SAwIN P. B., I947. Morphogenetic studies of the rabbit. XIX. Organ size in relation to body size in large race III and in small race X. Anat. Rec., 129, 457-472.

LATIMER H. B., SAwIN P. B., I962. Morphogenetic studies of the Rabbit. XXXI. Weights and linear measurements or some of the bones of 65 race III rabbits. Am. J. Anat., 110, 259-268.

I.ATIMER H. B., SAwIN P. B., I967. Morphogenetic studies of the Rabbit. XXXIX. Ponderal correlation coefficients of the bones from two races of rabbits. Anat. Rec., 159, 29-32.

OpIChal M., I950. Biometric analysis of the economic value of rabbits. Sborn. Čsl. Akad. Zemèd., 22, 344-363 (in Anim. Breed. Abstr., 18: 64r). 
Palkin G. A., 1946. Investigations on the composition of rabbits. Dokl. naùc Konf. Timirjazew. Seljskokoz. A kad., (4), 229-231 (in Anim. Breed. Abstr., 17: 157).

PIotrowicz Z., r967. The effect of crossbreeding on carcass quality in rabbits. I. Reciprocal cross between the Belgian Grey Giant and the Chinchilla. Acta agr. Silvest. Ser. Zootech. Krakov, 7, (I), 19-33 (in Anim. Breed. Abstr., 36: 4024).

PoDhrasky J., 1948. The influence of sex on the economic value of rabbits. Sborn čsl. Akad. Zemèd., 20, 312-32 I (in Anim. Breed. Abstr., 17: 561).

RICARD F. H., x965. Note sur l'héritabilité du rendement à l'abattage chez le poulet. Ann. Zootech., 14, $279-283$.

Ricaro F. H., Rouvier R., I966. Étude des mesures de conformation du poulet . IV. Vatiabilité génétique des mensurations de carcasse et d'un écart viande los chez des coquelets "Bresse-Pile". Ann. Zootech., 15, (2), I97-209.

RICARD F. H., Rouvier R., I967. Étude de la composition anatomique du poulet. I. Variabilité de la répartition des différentes parties corporelles chez des coquelets "Bresse-Pile". Ann. Zootech., 16, (r), 23-39.

RICARD F. H., Rouvier R., r969. Étude de la composition anatomique du poulet. III. Variabilité de la répartition des parties corporelles dans une souche du type Cornish. Ann. Génét. Sél. anim., 1, I 5 I-I 65.

RICARD F. H., Rouvier R., I970. Variabilité génétique du poids des parties corporelles chez le poulet. Application à la sélection. XIV Congrès Mondial d'Aviculture. Madrid (à paraître).

Rouvier R., RICARD F. H., I967a. Étude de la composition anatomique du poulet. II. Variabilité de la répartition de la viande et de l'os chez des coquelets "Bresse-Pile". Ann. Zootech., 16, 357-374.

ROUvier R., RICARD F. H., I967b. Recherche d'un index de sélection indirecte du poids de viande à poids d'os constant chez le poulet. Ann. Zootech., 16, r7-2r.

Rouvier R., I969. Variation des besoins d'entretien et de croissance chez les jeunes lapins ( 42 à 84 jours) de deux races. Ann. Génét. Sél. anim., 1, 197.

Shafie M. M., Badreldin A. I.., Ghany M. A., Hanafi M., I96r. Differential growth and carcass characteristics in the Giza rabbit. J. Anim. Prod. U.A.R., 1, I35-148.

VENGE O., 1953. Studies of the maternal influence on the growth in rabbits. Acta Agriculturae Scandinavica, 3, 243-29r.

Villegas V., 1958. Carcass values of rabbits. Philipp. Agric., 41, 538-54r.

YAO T. S., EATON O. N., 1954. Heterosis in the birth weight and slaughter weight in rabbits. Genetics, $39,667-676$. 